\title{
Reducing the Susceptibility of Lumped-Element KIDs to Two-Level System Effects
}

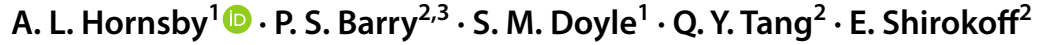

Received: 20 August 2019 / Accepted: 3 July 2020 / Published online: 23 July 2020

(c) The Author(s) 2020

\begin{abstract}
Arrays of lumped-element kinetic inductance detectors (LEKIDs) optically coupled through an antenna-coupled transmission line are a promising candidate for future cosmic microwave background experiments. However, the dielectric materials used for the microstrip architecture are known to degrade the performance of superconducting resonators. In this paper, we investigate the feasibility of microstrip coupling to a LEKID, focusing on a systematic study of the effect of depositing amorphous silicon nitride on a LEKID. The discrete and spatially separated inductive and capacitive regions of the LEKID allow us to vary the degree of dielectric coverage and determine the limitations of the microstrip coupling architecture. We show that by careful removal of dielectric from regions of high electric field in the capacitor, there is minimal degradation in dielectric loss tangent of a partially covered lumpedelement resonator. We present the effects on the resonant frequency and noise power spectral density and, using the dark responsivity, provide an estimate for the resulting detector sensitivity.
\end{abstract}

Keywords CMB $\cdot$ Instrumentation $\cdot$ Kinetic inductance detectors

\section{Introduction}

The temperature and polarization anisotropies contained in the cosmic microwave background (CMB) offer a unique window into how our Universe began. Detection of primordial B-modes is one of the main objectives for future CMB experiments and are critical to testing models of inflation $[1,2]$. Current experiments,

A. L. Hornsby

hornsbyal@cardiff.ac.uk

1 School of Physics and Astronomy, Cardiff University, The Parade, Cardiff CF24 3AA, UK

2 Department of Astronomy and Astrophysics, University of Chicago, 5640 South Ellis Avenue, Chicago, IL 60637, USA

3 Argonne National Laboratory, 9700 South Cass Avenue, Lemont, IL 60439, USA 
with focal planes based on arrays of superconducting transition-edge sensors (TESs), now routinely operate with sensitivity close to the backgroundlimit[3-5]. To further increase sensitivity, more detectors are required. It is now well known that observations at multiple frequencies are necessary in order to constrain and remove foreground contamination[3]. To maximize focal plane efficiency, each on-sky pixel includes dual-band, dual-polarization sensitivity, with each pixel requiring four detectors. The number of detectors required by the next generation of CMB experiments presents a significant technical challenge.

Kinetic inductance detectors (KIDs) are superconducting resonators whose resonant frequency and quality factor are modified with absorbed optical power[6]. Large arrays of KIDs can be constructed without the need for ancillary multiplexing components, significantly reducing the cryogenic complexity of an experiment. Currently, most existing CMB experiments implement an onchip microstrip optical coupling architecture, where radiation is guided onto a thin-film microstrip line and then routed to the detector, enabling multi-chroic, polarization-sensitive pixels. To take advantage of this heritage, and the separated inductive and capacitive elements of a lumped-element KID (LEKID), we have developed the microstrip-coupled LEKID (mc-LEKID)[7, 8].

The goal of the mc-LEKID is to provide a simple and reliable design for microstrip coupling to a LEKID. Here, the resonator inductor doubles as both a high-Q microwave lumped-element inductor, as well as an absorbing mm-wave microstrip line (Fig. 1, right). The microstrip line from the antenna is galvanically connected to the center of a hairpin-style inductor. Radiation incident along the input microstrip line is split and is absorbed along the length of the inductor. Feeding the inductor at the voltage-null enables a direct connection to the microwave resonator without affecting the performance. However, the microstrip dielectric is formed from an amorphous material that is deposited over the KID,

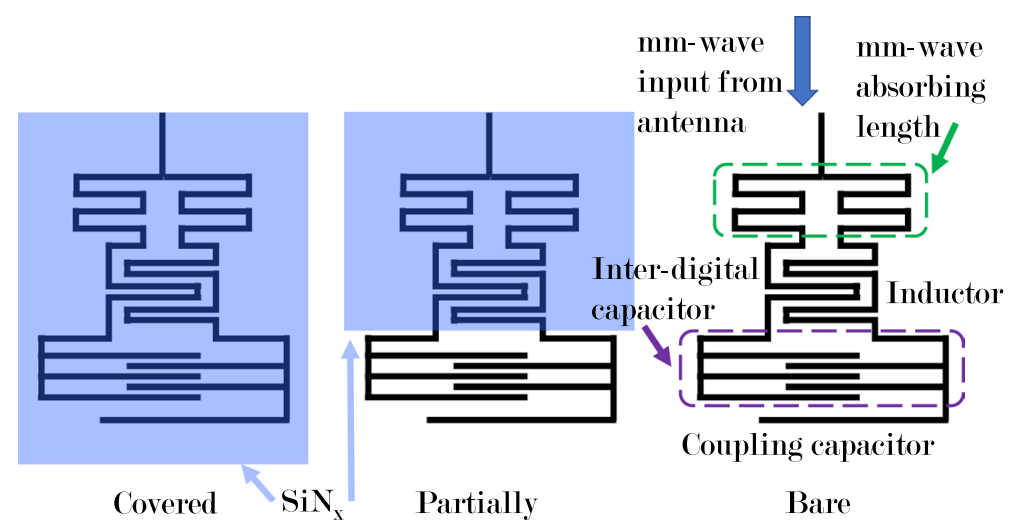

Fig. 1 Schematic describing the three different dielectric scenarios presented in this paper. $\operatorname{Left} \mathrm{SiN}_{\mathrm{x}}$ covers the entire resonator. Center: $\mathrm{SiN}_{\mathrm{x}}$ is over the inductor only. Right: Resonator free of dielectric. This addition of dielectric is necessary for transmission line coupling in antenna-coupled devices. (Color figure online) 
which has a tendency to degrade the performance of the KID resonator through the introduction of two-level systems (TLSs)[9-11].

In this paper, we investigate the effects on the microwave resonator caused by deposition of a silicon nitride $\left(\mathrm{SiN}_{\mathrm{x}}\right)$ dielectric layer over the LEKID architecture. In particular, we compare LEKID resonators with varying coverage of $\mathrm{SiN}_{\mathrm{x}}$ to study the impact on resonant frequency as a function of temperature and noise, as a first look at TLS contributions in a LEKID device architecture.

\section{Prototype Device}

The prototype device presented here is a single-layer, dark array of 18 thin-film aluminum (Al) LEKIDs, with various $\mathrm{SiN}_{\mathrm{x}}$ coverage to investigate the impact of adding the dielectric to our detectors. Figure 1 shows a schematic version of the devices and highlights our three different scenarios. Here, our resonators are grouped into three frequency banks, which span (i) $786-801 \mathrm{MHz}$, (ii) $1029-1058 \mathrm{MHz}$ and (iii) 1100-1166 MHz, each containing 6 resonators.

The lowest frequency resonators, bank (i), are completely covered by $\mathrm{SiN}_{\mathrm{x}}$ and are expected to have the highest loss (Fig. 1, left). The partially covered resonators (Fig. 1, center), bank (ii), have $\mathrm{SiN}_{\mathrm{x}}$ over the inductor only-this is the desired dielectric coverage for the proposed coupling scheme[8]. The highest frequency bank of resonators, bank (iii), is completely free of dielectric (Fig. 1, center). While this does not allow for optical coupling via a microstrip, this structure serves as a reference device for our proposed design.

During device fabrication, 50-nm layer of $\mathrm{Al}$ is first sputtered on to the silicon wafer to ensure maximum control over processing the $\mathrm{Al}$ which, in turn, reduces the number of TLSs created by the original substrate[12]. A wet etchant is used to pattern the $\mathrm{Al}$ film. Next, $500 \mathrm{~nm}$ of $\mathrm{SiN}_{\mathrm{x}}$ is deposited in a high-density plasma chemical vapor deposition system. To further reduce our susceptibility to TLSs, we fluorine-etch the $\mathrm{SiN}_{\mathrm{x}}$ from the different regions of the LEKID, depending on the desired level of dielectric coverage. For further details of the device fabrication process, see the $\mathrm{Al}$ and $\mathrm{SiN}_{\mathrm{x}}$ elements of Tang et al.[13].

\section{Results}

As a preliminary investigation into the impact of $\mathrm{SiN}_{\mathrm{x}}$ on the performance of the prototype device, we explored the dark response of the resonators outlined in Fig. 1. The prototype device sits in a gold-plated OFHC copper sample box installed on the $80 \mathrm{mK}$ baseplate of a cryostat, cooled via a miniature dilution system[14]. We measured $S_{21}$ of the resonators as a function of base temperature between 110-320 mK using a vector network analyzer (VNA), and extracted the resonant frequency following the procedure outlined in Khalil et al.[15]. As determined by the bifurcation point, each detector was driven at their optimal readout power to reach a minimum detector noise and this varied between detectors[16]. Generally, partially covered and covered resonators had a higher optimum drive power than bare resonators. 


\subsection{Dark Response}

We investigated the fractional frequency shift of each resonator as a function of base temperature, as the first measure of detector performance. In Fig. 2a-c, we observe the so-called back-bending in all devices, where the resonant frequency increases with temperature instead of decreasing, typical of TLSs perturbations[17-19]. Removing $\mathrm{SiN}_{\mathrm{x}}$ from the capacitive region of the LEKID reduces back-bending substantially; however, there is no discernible difference between the partially covered and bare resonator (Fig. 2b, c). Thus, the addition of $\operatorname{SiN}_{x}$ to the LEKID architecture does not introduce a significant additional TLSs frequency response relative to the completely bare resonator.

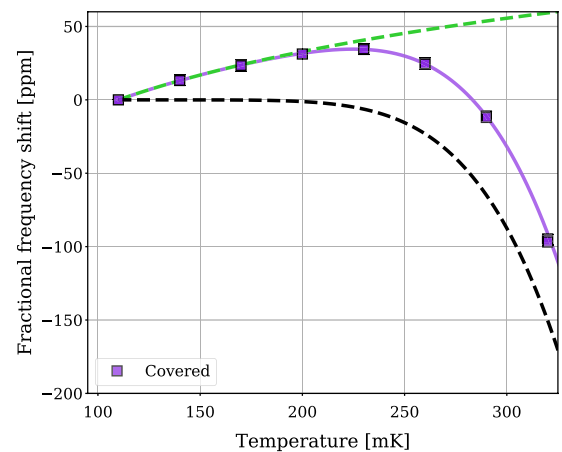

(a)

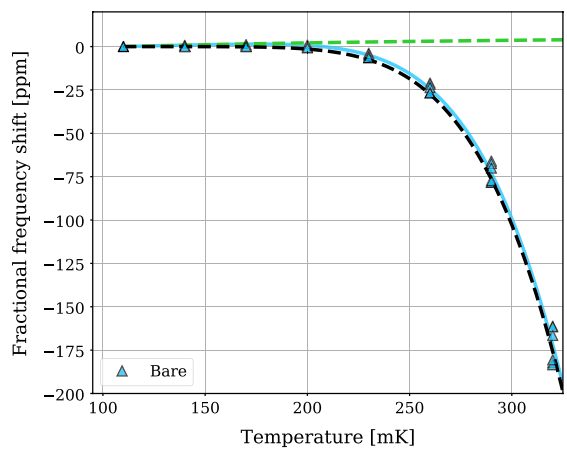

(c)

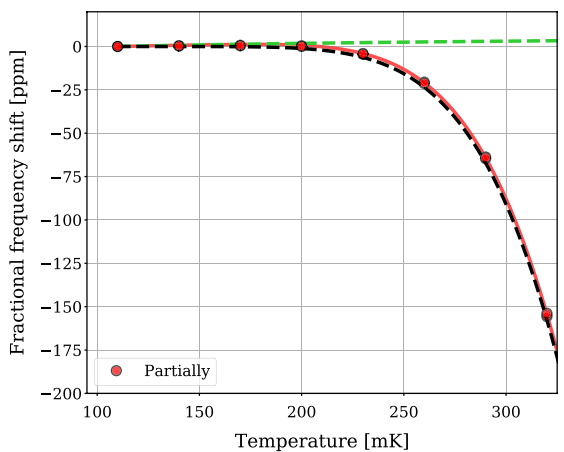

(b)

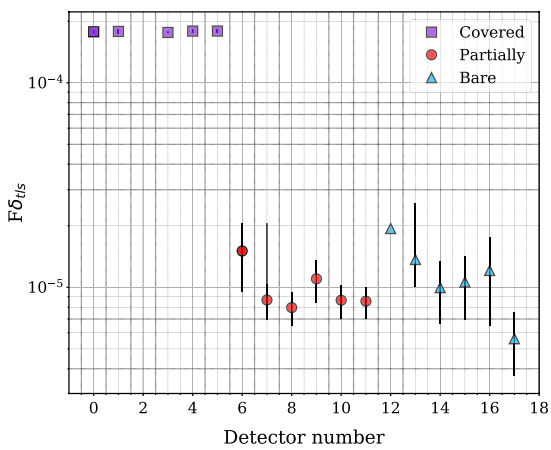

(d)

Fig. 2 The fractional frequency shift as a function of temperature for our resonators, as described in Fig. 1, where $\mathbf{a}$ is covered, $\mathbf{b}$ is partially covered and $\mathbf{c}$ is completely free of by $\mathrm{SiN}_{x}$. One covered resonator did not yield. The dashed lines are the two different components to the fit of the data-the expected fractional frequency shift (dashed black line) and the additional shift caused by TLSs, calculated from Eqs. 1 and 2 (dashed green line). The combined fit is a solid line. Plot $\mathbf{d}$ is the product $\mathrm{F} \delta_{0}$ extracted from fitting the fractional frequency response of each resonator, where the error bars indicate the error in fit. (Color figure online) 
We can begin to quantify the effect of adding lossy dielectrics to our resonators by considering the product of the fill factor and the TLS-induced loss tangent $F \delta_{0}$ [17-19].

$$
\frac{\Delta f_{r}}{f_{r}}=-\frac{F}{2} \frac{\Delta \epsilon}{\epsilon}
$$

where $\epsilon$ is the dielectric constant parametrized as,

$$
\frac{\Delta \epsilon}{\epsilon}=\frac{2 \delta_{0}}{\pi}\left[\operatorname{Re} \Psi\left(\frac{1}{2}+\frac{1}{2 \pi i} \frac{\hbar \omega}{k T}\right)-\log _{e} \frac{\hbar \omega}{k T}\right],
$$

$\omega$ is the frequency, $\Psi$ is the complex digamma function. Here, we fit a combined model of the additional frequency response from the dielectric and the expected response from Mattis-Bardeen superconductivity. From the fits (solid lines in Fig. $2 \mathrm{a}-\mathrm{c}$ ), we found removing the dielectric from the capacitive region of the LEKID, on average, reduced $F \delta_{0}$ from $(1.78 \pm 0.02) \times 10^{-4}$ to $(1.03 \pm 0.11) \times 10^{-5}$. On average, this equates to a reduction in $F \delta_{0}$ of $\sim 16$ across the partially covered and bare resonators of the prototype array. The average $F \delta_{0}$ was found to be $(1.14 \pm 0.18) \times 10^{-5}$ for the bare resonators. It should be noted that the capacitor geometry for each LEKID is very similar, meaning $\mathrm{F}$ should only vary slightly between detectors meaning this experiment provides good insight into the dielectric loss tangent.

\subsection{Dark Detector Noise}

To characterize the noise of the detectors and estimate the electrical noise equivalent power (NEP), we utilized the standard single-tone homodyne readout[6].

In Fig. 3a, we show the fractional frequency noise $\left(\mathrm{df} / \mathrm{f}_{0}\right)$ of each detector at a modulation frequency of $50 \mathrm{~Hz}$ to compare detector noise in the flat region of the noise power spectral density. Overall, we found the covered resonators had the highest noise level of all resonators with an average of $(3.81 \pm 0.20) \times 10^{-18} \mathrm{~Hz}^{-1}$, compared to the partially covered at $(1.53 \pm 0.16) \times 10^{-19} \mathrm{~Hz}^{-1}$ and the bare resonators at $(1.80 \pm 0.17) \times 10^{-19} \mathrm{~Hz}^{-1}$. Thus, the average noise power level is $\sim 25$ times higher compared to the partially covered resonator and $\sim 21$ times higher than the bare resonator, which indicates adding the dielectric to the absorbing element of the detector does not result in excess noise being observed. Moreover, this suggests that noisier dielectric materials could be used with this detector architecture, but this requires testing of additional devices in the future.

We estimate the responsivity of resonators 0,8 and 15 by fitting the fractional frequency response as a function of quasi-particle number (see Fig. 3b). Low temperature data points are excluded due to back-bending and the error shown here is the error in fit. With the fractional frequency noise level and detector responsivity calculated from the noise power spectral density (see Fig. 3c), alongside known material parameters, we can determine the dark NEP from Baselmans et al.[20]. The resulting NEP, derived from the responsivity and 


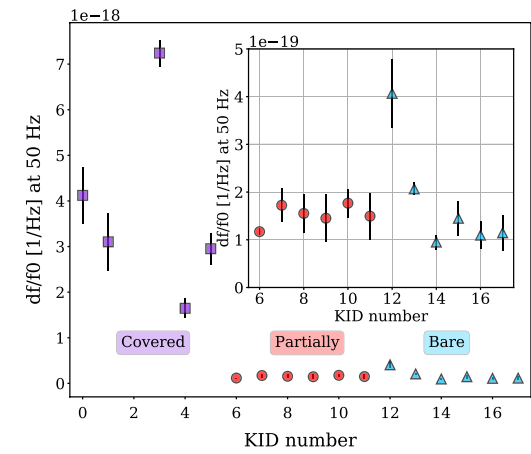

(a)

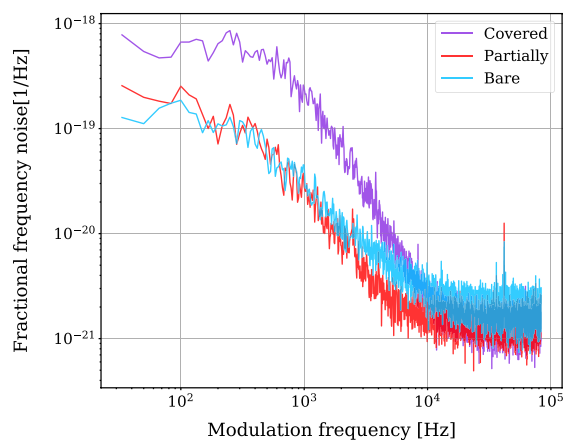

(c)

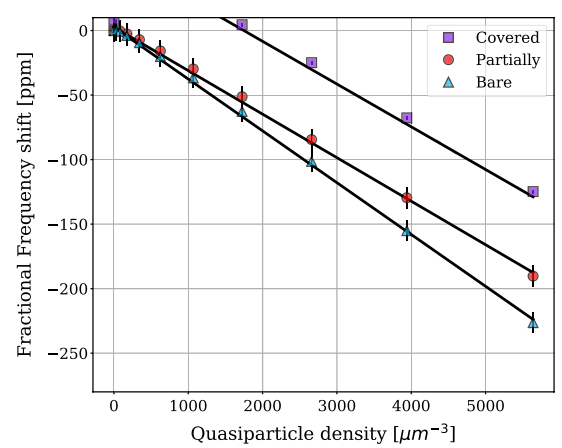

(b)

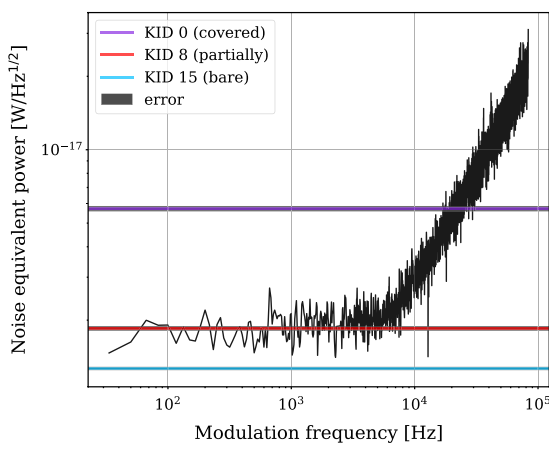

(d)

Fig. 3 a Measuring the fractional frequency noise at a modulation frequency of $50 \mathrm{~Hz}$ for covered (purple), partially covered (red) and bare (blue) resonators at $80 \mathrm{mK}$. One covered resonator did not yield. The inset in a allows for a closer look at $\mathrm{df} / \mathrm{f}_{0}$ for the bare and partially covered resonators. An average of several data points was used to determine the fractional frequency noise level at $50 \mathrm{~Hz}$, thus the error here represents the range of results, b Estimating the responsivity of a covered (purple), partially covered (red) and bare (blue) KID from the linear response of the resonator to the changing quasi-particle density as a function of base temperature. Only higher temperature data points are fitted due to the presence of back-bending, and the error shown is the extracted error in fit, $\mathbf{c}$ The measured noise spectrum of a covered (purple), partially covered (red) and bare (blue) resonator at $160 \mathrm{mK}$. d Dark noise equivalent power calculated (black) after the noise power spectral density of a partially covered resonator was corrected for the quasi-particle roll off. The measured dark NEP values for the covered (purple), partially covered (red) and bare (blue) are also shown here. (Color figure online)

corrected for quasi-particle roll off, is shown in Fig. 3d for the partially covered resonator 8, alongside the NEP calculated for resonators 0 and 15 . The NEP values are $(5.73 \pm 0.12) \times 10^{-18} \mathrm{~W} \mathrm{~Hz}^{-1 / 2}$ for the covered resonator (KID 0), $(1.85 \pm 0.03) \times 10^{-18} \mathrm{~W} \mathrm{~Hz}^{-1 / 2}$ for the partially covered resonator (KID 8) and $(1.28 \pm 0.02) \times 10^{-18} \mathrm{~W} \mathrm{~Hz}^{-1 / 2}$ for the bare resonator (KID 15).

To fully characterize the detector noise properties we need to measure the $1 / f$ knee of the noise spectrum. However, excessive system $1 / f$ noise is currently prohibiting low-frequency measurements. Going forward, the noise of each detector 
must be measured simultaneously to allow for de-correlated noise analysis and to enable the exploration of low-frequency behaviour.

\section{Conclusion}

Optical coupling to a LEKID via an antenna and transmission line structure is a promising candidate for future $\mathrm{CMB}$ experiments requiring large detector arrays, making possible the addition of structures needed for multi-chroic, polarizationsensitive capabilities. Separating the inductive and capacitive elements allows for additional TLS losses, caused by placing dielectric over the resonator, to be minimised. For a dark, single-layer LEKID array, we have demonstrated that we can meet the dielectric requirements of the microstrip transmission line coupling, while maintaining a minimal additional parasitic dielectric response and without creating additional noise.

Acknowledgements We acknowledge support from the Science and Technology Facilities Council (STFC) Consolidated Grant Ref: ST/N000706/1. This work is also supported by the National Science Foundation under Grant Number 1554565 and made use of the Pritzker Nanofabrication Facility of the Institute for Molecular Engineering at the University of Chicago, which receives support from SHyNE, a node of the National Science Foundation's National Nanotechnology Coordinated Infrastructure (NSF NNCI-1542205).

Open Access This article is licensed under a Creative Commons Attribution 4.0 International License, which permits use, sharing, adaptation, distribution and reproduction in any medium or format, as long as you give appropriate credit to the original author(s) and the source, provide a link to the Creative Commons licence, and indicate if changes were made. The images or other third party material in this article are included in the article's Creative Commons licence, unless indicated otherwise in a credit line to the material. If material is not included in the article's Creative Commons licence and your intended use is not permitted by statutory regulation or exceeds the permitted use, you will need to obtain permission directly from the copyright holder. To view a copy of this licence, visit http://creativecommons.org/licen ses/by/4.0/.

\section{References}

1. Planck Collaboration XXXVIII, A\&A 586, A141 (2016)

2. S.J. Huber et al., JCAP 1603, 036 (2016)

3. BICEP2/Keck Collaboration and Planck Collaboration, Phys. Rev. Lett. 114, 10 (2015)

4. B.A. Benson et al., Proc. SPIE 9513 (2014)

5. R.J. Thornton et al., ApJS 227, 21 (2016)

6. P.K. Day et al., Nature 425, 817-821 (2003)

7. S. Doyle et al., J. Low. Temp. Phys. 151(530), 536 (2008)

8. P.S. Barry, J. Low Temp. Phys. 193, 3 (2018)

9. W.A. Phillips, J. Low Temp. Phys. 7, 351 (1972)

10. P.W. Anderson et al., Philos. Mag. 25, 1 (1972)

11. J.A. Schlaerth et al., J. Low Temp. Phys. 167, 347 (2012)

12. E. Shirokoff et al., J. Low Temp. Phys. 176, 5 (2014)

13. Q.Y. Tang, J. Low Temp. Phys. 193, 3 (2018)

14. G. Teleberg, S.T. Chase, L. Piccirillo, J. Low Temp. Phys. 151, 669 (2008)

15. M.S. Khalil et al., JAP 111, 054510 (2012)

16. L.J. Swenson et al., J. Appl. Phys. 113, 104501 (2013) 
17. J. Gao et al., Appl. Phys. Lett. 92, 152505 (2008)

18. R. Barends et al., Appl. Phys. Lett. 92, 22 (2008)

19. R. Barends et al., Appl. Phys. Lett. 97, 3 (2010)

20. J. Baselmans et al., J. Low Temp. Phys. 151, 1 (2008)

Publisher's Note Springer Nature remains neutral with regard to jurisdictional claims in published maps and institutional affiliations. 